Nombres, combinaisons, probabilités

\title{
L'EXPÉRIENCE DES NOMBRES DE BERNARD FRENICLE DE BESSY
}

\author{
Catherine GoLDSTEIN
}

RÉsumé : Focalisé sur un problème posé par Bernard Frenicle de Bessy vers 1639, sa solution et les réponses de ses correspondants, cet article s'attache à décrire plusieurs registres enchevêtrés de l'expérience du mathématicien : expérimentation sur les nombres empruntée en partie aux sciences de la nature, injonctions d'une pratique collective cimentée par les problèmes et leurs constructions explicites, entrâ̂nement personnel de l'attention et du savoir-faire s'articulent ainsi dans les efforts de Frenicle pour contester la suprématie de l'analyse algébrique et dans les modes de conviction qui fondent son travail. Ce récit d'expérience permet en retour d'apprécier les problèmes que posèrent à Frenicle la transmission de ses résultats et, à ses historiens, la restitution de son activité.

Mots-ClÉs : Frenicle de Bessy, Fermat, Descartes, nombres, problèmes mathématiques, expérimentation, histoire sociale des mathématiques.

ABSTRACT : Through the discussion of a problem posed around 1639 by Bernard Frenicle de Bessy, its solution and the replies of his correspondents, we shall describe several overlapping registers of Frenicle's experience : an experimentation on numbers partly borrowed from the natural sciences, the demands of a collective practice cemented together by problems and their explicit construction, a personal training in attention and savoir-faire. All these come together in Frenicle's efforts to contest the supremacy of algebraic analysis and in the modes of conviction which are at the base of his work. In return, this account of a mathematical and collective experiment allows us to appreciate the difficulties for Frenicle in transmitting his results and for his historians in reconstructing his activity.

KEYWORDS : Frenicle de Bessy, Fermat, Descartes, numbers, mathematical problems, experimentation, social history of mathematics.

Revue de synthèse : $4^{\mathrm{e}}$ sér., ${ }^{\text {os }}$ 2-3-4, avr.-déc. 2001, p. 425-454. 
Zusammenfassung: Ausgehend von einem Problem, das Bernard Frenicle de Bessy ca. 1639 aufgeworfen hat, von seiner Lösung und den Antworten seiner Korrespondenten, beschreibt der Artikel mehrere, untereinander verschränkte Erfahrungshorizonte des Mathematikers : Zahlenexperimente, die z.T. den Naturwissenschaften entlehnt sind, Imperative einer kollektiven Praxis, die durch die Art der gestellten Probleme und expliziten Konstruktionen festgelegt ist, sowie Frenicles persönliches Schärfen seiner Aufmerksamkeit und seines Könnens. All dieses artikuliert sich in Frenicles Bemühen, die Überlegenheit der algebraischen Analysis zu bestreiten, ebenso wie in den Überzeugungsmodi, die seiner Arbeit zugrundeliegen. Im Gegenzug erlaubt die Erzählung der Mathematikererfahrungen ein besseres Verständnis der Probleme, die die Vermittlung seiner Ergebnisse für Frenicle aufwarf, und für den Historiker ein besseres Verständnis seines Wirkens.

STICHWÖRTER: Frenicle de Bessy, Fermat, Descartes, Zahlen, mathematische Probleme, Experiment, Sozialgeschichte der Mathematik.

Catherine Goldstein, née en 1958, est chargée de recherche au Centre national de la recherche scientifique, dans le cadre du laboratoire de mathématiques de l'université de ParisSud (UMR 8628). Elle cherche à développer une histoire sociale du travail et des résultats mathématiques, en particulier de la théorie des nombres en Occident. Elle est l'auteur de Un théorème de Fermat et ses lecteurs (Saint-Denis, Presses universitaires de Vincennes, 1995) et a codirigé avec Jeremy Gray et Jim Ritter, L'Europe mathématique. Histoires, mythes, identités (Paris, Éd. de la Maison des sciences de l'homme, 1996).

Adresse : CNRS et Université de Paris-Sud (UMR 8628), Bât. 425 Université de Paris-Sud, 91405 Orsay Cedex.

Courrier électronique : Catherine.Goldstein@math.u-psud.fr 
«M. Frenicle se tient fort caché », constate Pierre Fermat en août $1638^{1}$. Trois siècles et demi plus tard, nous ne pouvons qu'agréer : Bernard Frenicle de Bessy est surtout connu comme le moins connu des premiers membres de l'Académie des sciences ${ }^{2}$. Il fut pourtant un proche de Marin Mersenne, celui à propos duquel Fermat confiait à Gilles Personne de Roberval: «M. Frenicle m'a donné depuis quelque temps l'envie de découvrir les mystères des nombres, en quoi il me semble qu'il est extrêmement versé ${ }^{3}$. » La perplexité latente des historiens à la fin du $\mathrm{XIX}^{\mathrm{e}}$ siècle est bien résumée par Moritz Cantor: " D'autres recherches de Frenicle doivent avoir été connues confidentiellement, car, d'après les travaux publiés par l'Académie, la grande estime, que Fermat en particulier vouait à Frenicle, ne s'explique en aucune manière ${ }^{4}$. » $\mathrm{Au} \mathrm{xx}^{\mathrm{e}}$ siècle, cette perplexité ne s'est pas tant résolue en une nouvelle cohérence que dissipée dans l'indifférence quasi générale et l'éparpillement des recherches, et Frenicle paraît aussi introuvable qu'à Ernest Coumet la Révolution scientifique d'Alexandre Koyré. Une fois encore, il me semble, notre perception est brouillée par «les clartés incertaines de la Caractérisation ${ }^{5}$ ». Je voudrais suggérer ici quelques remèdes et surtout de bonnes raisons pour vouloir les chercher.

\section{FRENICLE DE BESSY ET LES EMBLÈMES DE LA PRATIQUE MATHÉMATIQUE AU XVII ${ }^{\mathrm{e}}$ SIÈCLE}

Frenicle n'a créé aucune théorie nouvelle, il n'a prouvé aucun théorème révolutionnaire; il n'a pas alimenté de commentaires théologiques ou philosophiques les grandes controverses qui marquent les débuts de la science moderne et n'en a pas non plus été la victime. Il était donc mal placé pour figurer parmi les savants héroïques. Mais si nous avons récemment quelque

1. Fermat, $O C$, vol. II, p. 165.

2. Exemple parmi d'autres, la présentation de STURDy, 1995, p. 98 : «Remarkably little information on the course of [Frenicle's] life has survived. [...] He is one of the more "elusive" founder-members of the Academy of Sciences even though he was just over sixty when it was founded. » L'information usuelle selon laquelle Bernard Frenicle de Bessy était conseiller à la Cour des monnaies n'est d'ailleurs pas fondée, voir CoumEt, [1968], vol. II, p. 554, et GolDSTEIN, 1995, p. 29.

3. Fermat, $O C$, vol. II, p. 203.

4. CANTOR, 1880-1908, vol. II, p. 784.

5. Coumet, 1987, p. 497. 
peu renoncé à eux, nous n'en avons pas fini avec les scientifiques représentatifs, de leur milieu, de leur temps ou de leur discipline - que l'identité que nous leur construisons soit celle, statistique, de l' «homme moyen » des démographes, ou un idéaltype à incarner, ou même, comme le propose Steven Shapin ${ }^{6}$, un simple matériau parmi d'autres dans le bricolage, au sens anthropologique, qu'est la légitimation d'une activité scientifique originale. Frenicle n'a guère laissé de métadiscours commodes qui suggéreraient au moins sa propre mise en scène et les opinions de ses contemporains, nuancées, ponctuelles, souvent contradictoires, se coordonnent mal en une caractérisation plausible. Sir Kenelm Digby, qui le fréquente lors de séjours à Paris, disqualifie d'ailleurs d'un coup la pertinence de trois références prometteuses, celles du docte, de l'humaniste et de l'honnête homme familier des salons : «J'ai dit à Monsieur Frenicle que, pour si grand feu d'esprit qu'il ait et quelque merveilleux que soit son génie pour la science des nombres, son feu seroit plus brillant, s'il le vouloit exciter ou augmenter par l'étude, par la lecture des anciens et par la conversation ${ }^{7}$. » Les embarras ne s'arrêtent pas au personnage social, et il est tout aussi délicat de résumer d'un trait les conceptions scientifiques de Frenicle, sa personnalité mathématique ou sa place dans le développement de la discipline. Expérimenté dans les nombres, Frenicle le fut sans doute, tous en témoignent, Pierre Fermat, Marin Mersenne, René Descartes, Claude Mylon, et Bernard Frenicle lui-même en tout premier lieu : «Il faudroit avoir bien peu de pratique aux nombres pour ne s'être pas aperçu d'abord que 7 est quatre fois la différence entre de fort petits nombres [l'un carré, l'autre double carré] savoir entre 1 et 8,2 et 9,18 et 25,25 et $32^{8}$. » Calculateur infatigable aussi, de carrés magiques dont il explicite dans un traité posthume les 880 cas de côté 4 , ou de triangles rectangles dont de larges tables subsistent dans les archives de l'Académie des sciences. D'où l'image classique d'un Frenicle amateur de nombres pour le plaisir de les manipuler, réduit aux mathématiques empiriques. Or, s'il y a bien expérience et calcul, nous aurons l'occasion de le constater, le mot «empirique » prête à confusion : tout comme Descartes, Frenicle a chapeauté ses grands traités, arithmétiques cette fois, par une méthode, La Méthode pour trouver la solution des problèmes par les exclusions, publiée à titre posthume en 1693, et qui, comme sa contemporaine plus célèbre, inclut une succession de procédures et des champs d'application.

«Le chercheur sérieux ne doit en aucune manière se détourner sans considération de ce que les amoureux des sciences de la sorte d'un Frenicle ont réalisé.

6. SHAPIN, 1991.

7. Fermat, $O C$, vol. II, p. 360.

8. Fermat, $O C$, vol. II, p. 238. 
De tels procédés - plutôt le fruit d'une forte habileté formelle — peuvent dans la main du maître devenir de grande importance pour la science ${ }^{9}$. »

Le compromis par lequel Joseph Hofmann concluait son étude du Solutio duorum problematum circa numeros cubos et quadratos..., le seul opuscule de Frenicle imprimé de son vivant, en 1657, lui assignait donc un second rôle dans la grande représentation du théâtre de la science. Parlonsnous du même auteur dont, en 1657 justement, Digby regrettait l'engouement soudain pour la théologie, qui risquait de faire perdre à Frenicle « le rang qu'il pourrait posséder d'un des plus grands mathématiciens du siècle $^{10} »$ ? Dont Fermat écrivait en 1643 : «Il n'y a certainement quoi que ce soit dans toutes les Mathématiques plus difficile que ceci, et hors M. de Frenicle et peut-être M. Descartes, je doute que personne en connoisse le secret ${ }^{11} \gg$ ?

De tels contrastes suggèrent autre chose que l'inadéquation de nos catégories routinières à caractériser Frenicle comme mathématicien : les limites de l'entreprise même de caractérisation. D'abord en ce que celle-ci doit postuler la stabilité des caractères qu'elle cherche à dégager et, dans la foulée, de leurs oppositions constitutives - empirique contre théorique, calcul contre démonstration, par exemple. Ensuite, en ce qu'elle leur assigne une valeur globale, et reste indifférente aux contextes de leur mise en évidence. Je voudrais adopter ici une approche inverse ${ }^{12}$, qui scrute l'expérience mathématique de Frenicle à travers ses usages mêmes, ses traces au cœur des textes, ses différences concrètes, opératoires, avec celle d'autres mathématiciens contemporains. Suivant les leçons de Coumet, qui examinait certains résultats combinatoires de Frenicle en transparence sur ceux de Mersenne $^{13}$, je me concentrerai sur un épisode particulier de la pratique arithmétique de Frenicle, un problème qu'il posa à Descartes et à quelques autres sur la construction d'un nombre fixé à l'avance d'ellipses dont certains segments sont mesurés par des entiers ${ }^{14}$. Circulant dans les correspondances, ce problème met en évidence la gamme des conceptions de l'activité arithmétique dans l'entourage de Frenicle. Dû à Frenicle lui-même, il

9. Hofmann, 1944, p. 40.

10. Fermat, $O C$, vol. II, p. 362.

11. Fermat, $O C$, vol. II, p. 248.

12. Malgré les différences évidentes de corpus, on pourra la rapprocher des propositions rassemblées in LEPETIT, dir., 1995, et REVEL, éd., 1996, en part. sur les problèmes d'identité, la distinction fondamentale entre singulier et local, la prise en compte explicite des contextes.

13. Coumet, [1968].

14. Le problème a été analysé par Paul Tannery dans son édition de Descartes, une analyse reprise en grande partie par les éditeurs de Mersenne, voir DesCaRTEs, $O C$, vol. II, p. 478-479, 509 et 541, ainsi que Mersenne, Correspondance, vol. VIII, p. 282-284, 309 et surtout l'appendice p. 744-746. Le manuscrit de Frenicle traitant de ce problème, en revanche, n'était alors pas disponible. 
permet d'apprécier le terrain propre où celui-ci souhaitait faire valoir ses ressources et son talent. Plusieurs modes d'expérience sur les nombres, relevant de registres dont nous avons maintenant une perception dissociée - disons, pour fixer les idées, personnel, collectif et technique - interfèrent en particulier dans les écrits de Frenicle, se définissent et se confortent mutuellement; les objections des mathématiciens contemporains aident à mettre en lumière leurs rôles et leurs articulations. De cet observatoire, il devient possible de recoller quelques-uns des fragments hétérogènes que j'ai évoqués plus haut, mais aussi d'apprécier ensemble le problème historique posé par la transmission d'une telle expérience et celui, historiographique, posé par sa description.

\section{LA QUESTION DES ELLIPSES}

\section{a) Les sources}

Entre fin 1638 et 1641 circula parmi les correspondants de Mersenne une « question des ellipses ${ }^{15}$ », posée par Frenicle. Il ne s'agit pas d'une question ouverte, mais d'un défi : l'auteur se devait donc en principe d'en connaître à l'avance une solution, le problème lui servant à éprouver les forces mathématiques de ses interlocuteurs ${ }^{16}$. À une exception tardive près, nous ne disposons d'ailleurs que de leurs réponses, celles de Florimond de Beaune (26 mars et 3 avril 1639), celles de Descartes, entre janvier et juin 1639, celle de Fermat le 25 décembre 1640, réponses à partir desquelles nous pouvons reconstituer un énoncé de la question - il est d'ailleurs possible qu'il ait varié quelque peu selon les interlocuteurs ou le moment : il s'agit de trouver un nombre (3, 4, parfois 12) fixé à l'avance d'ellipses de même grand diamètre, dont certains segments ont pour mesure des entiers et vérifient éventuellement certaines inégalités (par exemple, la distance focale doit être plus grande que le petit diamètre). Outre ces lettres, est maintenant connu un traité inédit de Frenicle, le Discours des triangles dont chaqu'un des trois cotés est un nombre entier ${ }^{17}$;

15. Fermat à Mersenne, in Fermat, $O C$, vol. II, p. 216; de Beaune à Mersenne, in MerSENNE, Correspondance, vol. VIII, p. 360.

16. Un autre cas contemporain, plus célèbre, celui de la cycloïde, est discuté in BEAULieu, 1982. Sur le fonctionnement et le rôle des défis arithmétiques dans la correspondance de Mersenne, voir Catherine Goldstein, « Numbers and letters. Arithmetical topics in Mersenne's correspondence », 2001, preprint.

17. Deux copies manuscrites (presque identiques) se trouvent dans les Archives de l'Académie des sciences. Je me servirai ici de celle conservée dans le fonds Roberval, carton 4, Discours des triangles... (cité par la suite comme Discours); le dernier chapitre commence p. $69 \mathrm{du} 4^{\mathrm{e}}$ cahier, le problème des ellipses est discuté plus particulièrement p. 78-81. Cet écrit a été identifié par Alan Gabbey, lors de son inventaire des papiers de Roberval, voir GABBEY, 
son dernier chapitre, consacré aux « lignes rationnelles contenues dans les ellipses » inclut une large portion du problème discuté ici. Frenicle y donne une nouvelle interprétation de la question, à laquelle Descartes et Mersenne font aussi allusion :

«Mais on pourrait deguiser en quelque facon ce probleme, \& le faire servir a la construction des verres Elliptiques que le $S^{r}$ Descartes employe a ses lunettes de courte vue. Il veut que la face du verre qui est au dedans du tuyau soit Elliptique, \& convexe, \& la face qui est au dehors soit circulaire, dont le centre soit le point brulant exterieur de l'Ellipse, lequel centre doit aussy etre la place de l'objet. On demande qu'une ligne rationnelle serve de grand diametre a tant d'Ellipses qu'on voudra, \& non plus, dont la surface convexe du verre sera portion, auxquelles le petit diametre soit moindre que la distance des points brulants, \& que tant le petit diametre de l'Ellipse, que l'epaisseur du verre, \& la distance de l'objet a iceluy soient lignes rationnelles ${ }^{18}$. »

Le problème des ellipses se situe ainsi à la confluence de deux phénomènes : d'une part, la réception dans le cercle de Mersenne des traités cartésiens de 1637, et plus spécifiquement de la Dioptrique ${ }^{19}$; d'autre part, l'intérêt dans ce cercle pour les problèmes de triangles en nombres, qui suscitèrent d'abondants échanges entre Fermat, Mersenne, André Jumeau de Sainte-Croix, Frenicle, puis Pierre Bruslart de Saint-Martin ${ }^{20}$. Diverses mentions manuscrites du problème par Mersenne ${ }^{21}$ confirment par ailleurs son importance dans l'environnement immédiat de Frenicle.

1966 et 1990. Je remercie les Archives de l'Académie des sciences de m'avoir permis de consulter et de citer des extraits de ce texte. Des informations supplémentaires sur l'édition posthume de fragments remaniés du Discours en tant que Traité des triangles rectangles en nombres en 1676 se trouvent in GoldSTEIN, 1995, en part. p. 37-40 et 149.

18. Discours, $4^{\mathrm{e}}$ cahier, p. $80-81$.

19. Plusieurs mathématiciens proches de Mersenne (Claude Mydorge, Girard Desargues, Blaise Pascal) écrivent d'ailleurs des ouvrages sur les coniques à peu près au même moment.

20. Voir la lettre du 16 décembre 1636 de Fermat à Roberval, in FERMAT, $O C$, vol. II, p. 93, pour une des premières mentions explicites de ce type de problème. Un triangle rectangle en nombres est un triangle rectangle dont les côtés sont mesurés par des nombres entiers, ou dans certains cas, fractionnaires : la relation de Pythagore ramène immédiatement ces questions à l'étude des relations $a^{2}=b^{2}+c^{2}$ pour des entiers (ou des rationnels) positifs $a, b$, $c$, en particulier à l'étude des propriétés des sommes de carrés. Sur les différents modèles pour ces questions avant l'entrée en scène de Frenicle et de Fermat, voir GoldsteIn, 1995, chap. XII, p. 123132.

21. Un manuscrit de Mersenne (Ms. BNF, NAF 5176) que ses éditeurs ont daté des années 1646-1647 contient une liste des chapitres du Discours des triangles ainsi qu'un résumé rapide d'une partie de son dernier chapitre, jusqu'au problème des ellipses. Mersenne a aussi consacré à ce problème quelques annotations en marge de son propre exemplaire de l'Harmonie universelle. L'ensemble a été édité (avec une erreur typographique dans l'exemple numérique) in Mersenne, Correspondance, vol. VIII, p. 282-283 et 744-753 respectivement. 


\section{b) Le problème}

La structure en étoile des échanges impose un examen séparé de chaque réaction au problème de Frenicle, et ce d'autant plus qu'elles ne sont pas vraiment comparables. Les protagonistes n'ont en effet ni les mêmes priorités mathématiques, ni la même hiérarchisation des problèmes et de leurs caractéristiques, ni les mêmes outils; leur communication est parfois entachée de malentendus, sur le sens à donner à un énoncé ou à une objection, sur la nature des solutions requises, et chaque intervenant interprète ici la tâche d'une manière différente, mettant en relief une dimension spécifique de la question de Frenicle.

De plus, même lorsqu'elle est parfaitement efficace pour eux, leur lingua franca mathématique n'est pas la nôtre. La norme adoptée en général pour les problèmes de nombres dans la correspondance de Mersenne consiste à expliciter la question posée sur un exemple numérique et à attendre en retour une réponse elle aussi numérique, ou au mieux une règle, c'est-àdire une procédure indiquant pas à pas comment fabriquer de telles solutions numériques. La méthode pour les obtenir, les justifications de la règle, en revanche, sont rarement communiquées, tant pour la fluidité des échanges que par manque de confiance interpersonnelle. Ce fonctionnement est d'ailleurs explicite, ainsi que ces variantes licites :

«Il me faudroit du temps, écrit Descartes à Mersenne en 1638, pour expliquer [ma méthode analytique] en forme d'une règle qui pust être entendue par ceux qui usent d'une autre méthode. Mais j'ay pensé que si je mettais ici une demidouzaine de nombres dont les parties aliquotes fassent le triple, vous n'en feriez peut-estre pas moins d'estat que si je vous envoyais une règle pour les trouver. [...] Les voicy ${ }^{22}$.»

Afin de simplifier la présentation, je commencerai par exposer brièvement certains éléments du problème qui apparaîtront de manière dispersée dans les discussions ultérieures. Je n'adhérerai pas ici aux textes originels, mais je ne prétends pas non plus décrire une quelconque essence du problème ou de sa solution; il ne s'agit que de disposer d'une armature commune qui permette de localiser les uns par rapport aux autres les fragments de solution dont nous avons hérité. Comme les mathématiciens concernés, je confondrai souvent les segments et leurs mesures. Je fixerai aussi dans tout ce qui suit une seule figure et les notations associées; celles-ci changent non seulement d'auteur à auteur, mais d'une lettre à l'autre, et même, chez Frenicle par exemple, à plusieurs reprises dans le même texte. Voici cette figure; $A B$ et $C D$ sont le grand et le petit diamètre de l'ellipse,

22. Mersenne, Correspondance, vol. VII, p. 345. 
$F$ et $F$ ' les deux foyers (également appelés à cette époque "points brûlants »), $H$ est le point d'intersection de l'ellipse et de la perpendiculaire au grand diamètre passant par le foyer $F, H O$ est un arc du cercle centré en $F$, et de rayon $F^{\prime} H$.

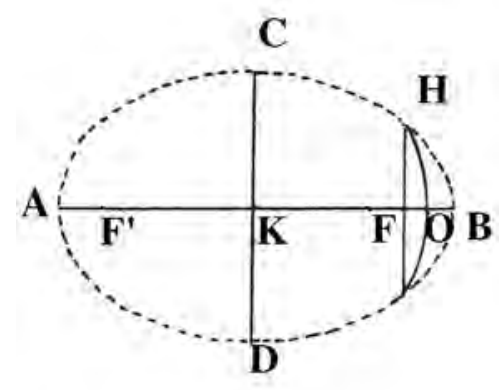

Selon la propriété caractéristique de l'ellipse, la somme des distances de tout point de l'ellipse aux deux foyers $F$ et $F$ ' est constante, et donc égale au grand diamètre $A B$ de l'ellipse. En appliquant cette propriété au point $C$, il vient $F^{\prime} C+F C=2 F C=A B$. Puisque le triangle $C K F$ est rectangle, on en déduit que $A B^{2}=F F^{\prime 2}+C D^{2}$ et on a donc associé à toute ellipse un triangle rectangle fondamental. Réciproquement, à partir d'un triangle rectangle et de la relation de Pythagore entre les côtés, on peut former deux ellipses, selon qu'on identifie la distance focale et le petit diamètre respectivement à l'un ou à l'autre des côtés de l'angle droit. On peut de plus prouver facilement que $2 H F$ est troisième proportionnelle avec le grand et le petit diamètre, autrement dit que $2 H F . A B=C D^{2}$. Toutes ces relations se démontrent à l'aide de la propriété caractéristique de l'ellipse appliquée à divers points et de relations classiques dans les triangles rectangles; elles sont indépendantes de la nature des nombres mesurant les segments considérés.

Frenicle, quant à lui, se limite au cas où certains segments sont entiers : les deux diamètres, la distance focale et le segment $H F$, ou bien, alternativement, selon les énoncés, les deux diamètres et les segments $O B$ et $O F$, ou bien encore, les deux diamètres, $F O$ et $B F$ - il est facile de montrer que tous ces problèmes sont équivalents. Le «triangle rectangle fondamental » de côtés $A B, C D, F F$ ', associé à l'ellipse, est alors un triangle rectangle en nombres entiers et la relation $2 H F . A B=C D^{2}$ entraîne que $A B$ et $2 H F$ sont, à un diviseur entier commun près, des carrés. Puisqu'un diviseur entier commun à $A B$ et à $H F$ est aussi un diviseur de tous les segments considérés, le seul diviseur un peu délicat à gérer est donc 2 et certains phénomènes dépendent effectivement de la parité du grand diamètre. Dans tous les cas, on peut prouver que $A B$ est divisible par un carré impair 
qui représente une hypoténuse de triangles rectangles, et dont la racine est encore une hypoténuse ${ }^{23}$. Réciproquement, posons pour clarifier $A B=d w^{2}$, où $w$ est un nombre impair, hypoténuse d'un triangle rectangle à côtés entiers, c'est-à-dire vérifie une relation $w^{2}=w^{\prime 2}+v^{2}$ pour certains entiers $v$ et $w^{\prime}$. On reconstruit alors facilement une ellipse adéquate, en posant :

$$
A B=d w^{2}, C D=d w w^{\prime}, F F^{\prime}=d w v .
$$

On a alors :

$2 H F=d w^{\prime 2}, 2 H F^{\prime}=d\left(2 w^{2}-w^{\prime 2}\right), 2 O F=d(w-v)^{2}, 2 F B=d w(w-v)$.

En particulier, si $d$ est pair, toutes ces lignes sont entières, ce qui veut dire que la décomposition $w^{2}=w^{2}+v^{2}$ fournit deux ellipses solutions (en échangeant le rôle de $w^{\prime}$ et de $v$ ); si $d$ est impair, une seule des deux ellipses possibles a tous les segments requis entiers, celle pour laquelle $w$ ' dénote le côté pair. Les conditions supplémentaires parfois ajoutées par Frenicle sur les ellipses-solutions reviennent à imposer que $v>w^{\prime}$ ou que $v^{2}<w w^{\prime}$. Le nombre d'ellipses cherchées de diamètre donné $A B$ se déduit alors du nombre de décompositions de $A B$ sous la forme $d w^{2}$ où $w$ est l'hypoténuse d'un triangle rectangle à côtés entiers vérifiant ces conditions.

Des différents aspects du problème - relations géométriques dans l'ellipse, rapport à la construction de triangles rectangles ou à un autre cadre théorique, caractérisation de la forme adéquate des diamètres, gestion de la condition d'intégralité des segments et des éventuelles inégalités supplémentaires, dénombrement exact des diviseurs pertinents du grand diamètre commun aux ellipses cherchées —, les mathématiciens impliqués, nous allons le voir, ont perçu de manière variée l'intérêt, la difficulté et la relation réciproque.

TROIS RÉPONSES ET QUELQUES ENJEUX

\section{a) La réaction de Beaune et le statut des problèmes sur les nombres}

Éclairant d'emblée l'enjeu du problème dans l'environnement de Mersenne, la réponse la plus radicale vient de Beaune; il l'exécute en ces termes, dans une lettre à Mersenne du 26 mars 1639 :

«Je vous supplie de me dispenser de la recherche de ceste question, pour m'apliquer, aux heures de mon loisir, a de plus serieuses: cette question

23. Ce résultat est difficile; il repose sur la détermination des nombres entiers qui sont hypoténuses de triangles rectangles en nombres (ou somme de carrés) et celle de leurs diviseurs, voir, p. ex., WeIL, 1984, chap. II. Nous en reparlerons. 
n'estant d'aulcun usage et ne tombant poinct soubs la science des rapports, qui les considere universelement, aussi bien entre les lignes commensurables et incommensurables, si bien que la recherche en seroit extremement laborieuse et de nul proffict, ce qui n'arrive pas en celles de geométrie et celles d'arithmetique qui tombent soubs ceste science des proportions, les aultres estant de peu de consideration et n'estant d'aulcun usage ${ }^{24}$.

Au détour d'une lettre ultérieure, début avril, il renchérit sur le thème : «La science doibt regarder les affections universelles et non pas s'attacher à une particulière ${ }^{25}$. » Un tel rejet est d'autant plus spectaculaire que le refus de se pencher sur un problème est rare dans la correspondance de Mersenne : proposer une réponse, même incomplète ou superficielle, est la garantie de son fonctionnement même comme lieu d'échanges mathématiques, et plusieurs correspondants font explicitement allusion à cette convention.

Je ne réexaminerai pas ici les relations entre «science » et « universel » au XVII ${ }^{\mathrm{e}}$ siècle $^{26}$. Ce qui m'intéresse davantage dans la déclaration de de Beaune, c'est son efficacité pour disqualifier un ensemble de questions arithmétiques (outre celle-ci, d'autres portant sur les parties aliquotes subissent le même sort) : dans leur sujet, dans leur objectif et dans leur pratique tout ensemble. Dans ce deuxième quart du XvII ${ }^{e}$ siècle, un outil privilégié de la science universelle des rapports que réclame de Beaune est bien sûr l'analyse algébrique, susceptible d'unifier les deux disciplines mathématiques théoriques traditionnelles, la géométrie et l'arithmétique ${ }^{27}$. $\mathrm{Au}$ cours de cette unification, le corpus des problèmes jugés intéressants est remodelé. Rejetées ainsi hors de la science, les recherches sur les entiers apparaissent à de Beaune aussi vaines qu' « extremement laborieuse[s] », en ce qu'elles ne relèvent pas d'une méthode systématique, propre selon lui à l'universel, mais d'un long et fastidieux travail, nécessaire pour parvenir au cas visé, et limité à lui. Ces critiques ne sont pas idiosyncrasiques, et les caractères attribués à la pratique mathématique sont parfois étendus au mathématicien même. Ainsi, Descartes à Mersenne, en 1638 :

«Pource qu[e les questions d'arithmétique] peuvent quelquefois mieux estre trouvées par un homme laborieux qui examinera opiniastrement la suite des nombres, que par l'adresse du plus grand esprit qui puisse estre, \& que d'ailleurs elles sont tres inutiles, je fais profession de ne vouloir pas m'y amu$\operatorname{ser}^{28}$.»

24. Mersenne, Correspondance, vol. VIII, p. 360.

25. Mersenne, Correspondance, vol. VIII, p. 372.

26. Voir, en part., dans le contexte d'une comparaison avec le point de vue de Frenicle, Eamon, 1994, p. 53-57, et Dear, 1995, p. 11-25.

27. Voir, p. ex., les textes analysés in Juhel, 1994, et Bos, 1996.

28. Descartes, $O C$, vol. II, p. 91. 
Non seulement la patience est ici requise, plus que la subtilité d'esprit ou la puissance méthodique, mais elle semble parfois suffisante, les entiers pouvant être testés un par un, jusqu'à trouver celui qui satisfait la question posée. Resituée dans ce débat, plus vaste et plus durable, la question de Frenicle apparaît comme un instrument de lutte : destinée comme nous le verrons à mettre en évidence les limites de l'algèbre, elle doit aussi manifester que son auteur ne cède pas de terrain sur la qualité des problèmes ou sur la nécessité de la méthode. Mersenne témoigne encore de ce contexte polémique, lorsqu'il conclut ses annotations sur ce problème par : «L'analyse n'a peu soudre ceste question jusques à présent ${ }^{29}$. »

\section{b) La réponse de Descartes : espoirs et limites de l'algèbre symbolique}

Nous possédons quatre lettres de Descartes sur le problème des ellipses, datant des six premiers mois de 1639. Pourquoi Descartes a-t-il accepté de réfléchir sur cette question, contrairement à de Beaune, dont il partage pourtant l'opinion sur les problèmes arithmétiques? «A cause que le Problesme que vous proposez concerne la Dioptrique, écrit-il dans sa première réponse à Frenicle, je pense estre obligé de faire de mon mieux pour le résoudre ${ }^{30}$. » Cela ne doit pas être interprété comme un jugement favorable sur la portée pratique du problème : Descartes reconnaît seulement que la question, retaillée sur mesure, lui est directement adressée et que la courtoisie apparente du procédé l'engage à répondre. Il cherche alors à se ménager des alliés à Paris; dans les mois précédents, à l'occasion d'autres problèmes arithmétiques portant sur les sommes de carrés et sur les parties aliquotes, il a reçu l'approbation de ses interlocuteurs, en particulier de Jumeau de Sainte-Croix; il a donc des raisons d'espérer encore un échange pacifique et même profitable à la promotion de son algèbre.

Dans les deux premières lettres, Descartes propose comme on s'y attend des règles, illustrées numériquement, pour construire le diamètre commun aux ellipses cherchées et toutes leurs lignes entières. Des critiques variées de Frenicle ${ }^{31}$ et l'insistance de Mersenne le contraignent finalement à dévoiler sa méthode. L'ensemble éclaire nettement les possibilités et les limites de l'algèbre symbolique telle que la plupart des analystes du

29. Mersenne, Correspondance, vol. VIII, p. 283.

30. Descartes, $O C$, vol. II, p. 471-477.

31. Nous ne les connaissons que par la réponse qu'y apporte Descartes, $O C$, vol. II, p. 537-538, et certaines semblent absurdes; elles font pourtant sens, malgré la maladresse apparente de la formulation, dès qu'on prend en compte l'économie du problème chez Frenicle. Faute de place, je ne pourrai malheureusement pas toutes les détailler ici, voir cependant infra, n. 72. 
$\mathrm{XVII}^{\mathrm{e}}$ siècle la font opérer sur les problèmes de nombres, et du même coup permet d'identifier un des lieux privilégiés où peuvent s'exercer les critiques des mathématiques cartésiennes.

La première suggestion de Descartes pour trouver le diamètre commun aux ellipses est d'élever au carré le plus petit commun multiple de nombres qui sont «milieux de nombres carrés » comme 5 (situé au milieu entre 1 et 9), 13 (entre 1 et 25), 25 (entre 1 et 49) et de multiplier par 4 le résultat; pour trois ellipses, il vient ainsi $422500\left(=4 .(25.13)^{2}\right)$. Sa deuxième suggestion, après une première critique de Frenicle dont nous ignorons le détail, est de multiplier entre eux autant de nombres premiers de la forme $n^{2}+1$ qu'on demande d'ellipses ${ }^{32}$ et d'élever au carré le résultat (à un facteur 3 près dont il avouera l'inutilité dans la lettre suivante), soit un diamètre de $(17.37)^{2}$ pour la construction de deux ellipses satisfaisant les conditions données.

Descartes relie donc bien à chaque fois le nombre d'ellipses cherchées à la décomposition du diamètre en facteurs d'un certain type, et l'intégralité requise des segments à une élévation au carré - nous verrons pourquoi lorsqu'il explicitera sa méthode - mais le type de facteurs qu'il met en évidence est plus restreint qu'une somme générale de carrés : les nombres «milieux de carrés » s'écrivent par exemple $k^{2}+(k+1)^{2}$, c'est-à-dire comme une somme de deux carrés consécutifs; quant à sa deuxième proposition, elle fait intervenir les sommes d'un carré et de 1 . Rien n'indique d'ailleurs que Descartes ait perçu à ce moment la possibilité d'utiliser des sommes de carrés générales pour construire les ellipses ${ }^{33}$.

Or, le principal obstacle à ces choix, comme il est bien connu, concerne le nombre exact d'ellipses obtenues: Descartes a apparemment espéré le contrôler d'abord en éliminant des facteurs parasites communs aux constituants du diamètre par le choix d'un p.p.c.m., puis en se limitant à des constituants premiers. Mais le produit de deux sommes de carrés, même de la forme particulière choisie par Descartes, comme $17=1+16$ et $37=$ $1+36$ par exemple, fournit non pas deux hypoténuses de triangles rectangles, mais quatre, chacune susceptible de fournir une ellipse adéquate à

32. La question de l'existence de nombres premiers de cette forme en aussi grand nombre qu'on veut (une question toujours ouverte en juillet 2000) n'est pas posée, mais la difficulté d'en trouver explique sans doute pourquoi Descartes se restreint ici à deux et non plus à trois ellipses comme auparavant.

33. Au contraire : la particularité des nombres utilisés est encore à l'œuvre dans les règles fournissant les autres segments dans chacune des trois ellipses, ces règles ne coïncidant avec celles déduites des formules générales données plus haut qu'à condition de supposer partout $w-v=1$; or, il est alors usuel d'expliciter, quand on donne une règle, les nombres qui, du strict point de vue du calcul de l'exemple choisi, ne comptent pas (une multiplication par 1, par exemple), ce qui suggère que Descartes n'a alors dérivé les règles que dans le cas où les diamètres ont la forme proposée. 
segments entiers ${ }^{34}$; les restrictions implicitement imposées par Descartes sur la forme des facteurs du diamètre — et qui ne sont de toute façon pas stables par multiplication — bloquent ici la maîtrise complète du dénombrement.

Les commentaires de Descartes aux objections de Frenicle sur ce point concordent de manière frappante avec la nature des réticences sur les problèmes de nombres que nous avons rencontrées chez de Beaune. «Il se peut faire que je me seray mepris en ma response a la question de $\mathrm{Mr}$ de Bessy, écrit-il d'abord, car l'ayant trouvee fort promptement par mon calcul, je ne m'arrestay presque point a en considerer les divers cas ${ }^{35} \gg$ : sont donc encore opposées ici la (lente) discussion des cas, des situations particulières, et l'efficacité du calcul algébrique qui traite «promptement » une partie de la question, du coup considérée comme la seule digne d'attention. Ce qui reste ne lui semble pas relever d'un cadre général qu'il s'agirait d'élaborer, mais d'exceptions, qui ne peuvent être contrôlées qu'individuellement, mais qui du même coup offrent peu d'intérêt :

«Ayant trouve tout d'abord tout ce qui me semblait contenir de difficulté en la question, qui estoit de donner autant d'ellipses rationelles qu'on voudroit qui eussent une mesme ligne pour plus grand diamètre, et ayant d'autres pensées dans l'esprit ${ }^{36}$, je ne me suis pas arresté a considérer toutes les exceptions qu'il falloit faire, afin que cette ligne ne servist point a plus grand nombre d'ellypses qu'a celuy qui seroit demandé ${ }^{37}$. »

Comment Descartes a-t-il donc trouvé «tout ce qui [lui] semblait contenir de difficulté »? Comme il l'indique à Mersenne le 30 avril $1639^{38}$, en choisissant comme inconnues principales $a$ la ligne $O F$ et $b$ la ligne $B F$, Descartes obtient les formules

$$
\begin{aligned}
& A B=2 \frac{b b}{a} \\
& C D=2 b \sqrt{\frac{2 b}{a}-1}
\end{aligned}
$$

34. D'hypoténuse 629, on trouve, outre les triangles évidents de petits côtés 37.15 et 37.8 , d'une part, 17.12 et 17.35 d'autre part, les deux triangles de côtés respectifs 100 et 621 , d'une part, 460 et 429 d'autre part. Notons que les ellipses correspondantes ne vérifient pas nécessairement les conditions d'inégalité supplémentaires; ici, trois d'entre elles seulement auraient une distance focale supérieure au petit diamètre.

35. Descartes, $O C$, vol. II, p. 506.

36. Nous voyons apparaître ici le thème classique de l'attention.

37. Descartes, $O C$, vol. II, p. 536.

38. Descartes, $O C$, vol. II, p. 536. 
Comme à d'autres occasions, Descartes compte sur un choix habile des inconnues pour exprimer confortablement la plupart des conditions du problème : ici, en effet, l'intégralité de deux des lignes requises s'énonce directement sur les inconnues ( $a$ et $b$ ), et les deux suggestions faites par Descartes correspondent à deux choix très simples pour assurer l'intégralité des deux diamètres, en supposant $a=1$ ou 2. Descartes ramène donc « fort promptement» le problème à des équations, qui sont universelles dans le sens où elles expriment les relations entre les lignes considérées, indépendamment de leur nature. Dans la situation en question, elles donnent même accès à « autant d'ellipses [...] qu'on voudroit de même diamètre ». Autant, mais non «autant... et non plus». Si cette démarche exprime des solutions, elle ne permet ni de reconnaître en général lesquelles exactement sont entières ${ }^{39}$, ni surtout de les énumérer précisément.

Descartes reconnaît d'ailleurs dans sa dernière lettre que le diamètre qu'il avait proposé possède des décompositions supplémentaires et il suggère :

«Il me semble que la meilleure solution est de faire que $A B$ soit un nombre carré impair dont la racine ou ses parties se puisse diviser en deux carrés, autant de fois qu'on demande d'ellipses [...]. Mais je ne voy pas qu'il soit aisé de donner reigle pour trouver un nombre qui se divise ainsy, luy ou ses parties, en autant de carrés qu'on voudra et non plus, si ce n'est qu'apres en avoir trouve autant qu'il faut, on en oste ceux qui s'y trouveront de plus en tastonnant. Il m'enseignera, s'il luy plaist, si je me trompe.»

La seule alternative proposée est donc le tâtonnement : l'intervention de Frenicle portera sur ce point, et en particulier sur l'impossibilité de contrôler le dénombrement demandé (avec les conditions d'inégalité supplémentaires) pour un diamètre commun impair, ce qui apparaît à Descartes une particularisation supplémentaire et artificielle du problème.

«Je n'avais plus envie de répondre [à Frenicle], conclut Descartes en juin 1639, car sa question n'est ni belle ni industrieuse. [...] mais je ne veux point contester, car il me paroist estre [...] du nombre de ceux qui veulent, a quelque prix que ce soit, avoir gaigne $\&$ parler les derniers ${ }^{40}$.»

Voici donc Frenicle en tâtonnant dogmatique, rajoutant des conditions pour tester la patience, et non la subtilité, de ses interlocuteurs; nous examinerons un peu plus loin l'autre face de cet échange.

39. Cette conception de la notion de généralité pour les solutions des équations algébriques se rencontre, p. ex., chez Lagny à la fin du siècle, voir GoldSTeIN, 1995, p. 173.

40. Descartes, $O C$, vol. II, p. 567. 


\section{c) La réponse de Fermat: pour une théorie des nombres}

Le détour par Fermat est néanmoins nécessaire, et il est d'autant plus intéressant que c'est son point de vue qui a prévalu chez les théoriciens des nombres postérieurs et, par conséquent, chez les historiens du domaine en particulier, c'est celui-ci qu'adoptent implicitement les éditeurs de Descartes et de Mersenne. C'est à la Noël 1640 que nous rencontrons la question des ellipses dans la correspondance de Fermat; le moment est crucial pour les relations de ce dernier avec Frenicle. Dès 1636, Fermat annonçait à Roberval une méthode générale pour «soudre par algèbre ${ }^{41}$ » les problèmes sur les nombres; il constituait donc une cible de choix pour les arithméticiens $^{42}$ parisiens. De fait, il a essuyé les questions de Jumeau de Sainte-Croix de 1638, proposé des solutions, lancé ses propres défis. Début 1640, à l'occasion d'un échange sur les carrés magiques, Frenicle tente de rétablir sa position auprès de Mersenne en traitant avec mépris les résultats de Fermat : «Pour Monsieur de Frenicle, réplique Fermat, ses inventions en Arithmétique me ravissent et je vous déclare ingénument que j'admire ce génie qui, sans l'aide de l'Algèbre, pousse si avant dans la connoissance des nombres entiers ${ }^{43}$. » Et de déployer en retour une manne de résultats et plusieurs questions. Les tests se poursuivent ainsi pendant plusieurs mois : ils conduisent à une confiance réciproque, fragile certes, mais qui permet des échanges directs, plus approfondis, contenant non seulement des défis et des solutions numériques, mais aussi des questions ouvertes, des demandes d'éclaircissements et de compléments, parfois des principes ou des indications de méthode. Le 25 décembre 1640, Fermat communique ainsi ses «fondements » sur le sujet de triangles rectangles :

« Tout nombre premier qui surpasse de l'unité un multiple du quaternaire, est une seule fois la somme de deux carrés, et une seule fois l'hypoténuse d'un triangle rectangle [...]. Ce même nombre étant une fois l'hypoténuse d'un triangle rectangle, son quarré l'est deux fois, son cube trois, son quarréquarré quatre, etc. à l'infini ${ }^{44}$. »

Fermat ajoute une règle pour déterminer combien de fois exactement un nombre donné est hypoténuse d'un triangle rectangle ${ }^{45}$ et conclut :

«Pour la question des ellipses, elle se déduira fort aisément de ce que vous venez de voir, car la question va là à trouver un nombre qui serve d'hypo-

41. Fermat, $O C$, vol. II, p. 93.

42. J'emploie ce mot non dans le sens d'une activité professionnelle — «professeurs d'arithmétique », - mais en reprenant le terme même de Mersenne pour désigner SainteCroix ou Frenicle.

43. Fermat, $O C$, vol. II, p. 187.

44. Fermat, $O C$, vol. II, p. 214.

45. Pour les résultats mathématiques en question, voir, p. ex., WeIL, 1984, chap. II. 
ténuse à 12 triangles et non plus [...]. Ce qui est aisé, et ayant trouvé le dit nombre, son quarré sera le demi-diamètre des ellipses. Il le faut quarrer, afin que la perpendiculaire sur le foyer soit un nombre entier. J'en dis assez pour me faire entendre à $\mathrm{M}$. Frenicle ${ }^{46}$. »

D'emblée, le problème des ellipses est donc pour Fermat recadré dans les recherches sur les triangles rectangles en nombres, dont il découle «fort aisément ». Le cœur du problème est la détermination et l'énumération exactes des nombres (appelés plus haut $w$ ) qui entrent dans la composition du grand diamètre, interprétés immédiatement comme hypoténuses de triangles rectangles en nombres entiers. C'est pour cette question que Fermat énonce une règle, se contentant d'une rapide (et incomplète) indication pour la détermination des segments de l'ellipse. La routine, non seulement la sienne propre, mais celle qu'il imagine commune à lui et à Frenicle au moins, en l'occurrence l'établissement des relations entre les segments, correspond donc à peu près, au contraire, à ce qui avait retenu l'attention de Descartes.

Fermat aborde ces questions dans la perspective d'une «science des nombres » (plusieurs fois annoncée, mais qu'il ne rédigera apparemment jamais), c'est-à-dire d'une élaboration ordonnée à partir de «fondements » comme ceux mentionnés plus haut. Ses intérêts arithmétiques personnels se concentrent d'ailleurs peu à peu sur la représentation des nombres comme somme de carrés et de multiples fixes de carrés, au détriment des thèmes classiques, comme les triangles rectangles en nombres, qui n'apparaissent plus que comme dérivés ${ }^{47}$. De plus, si la résolution de problèmes reste au premier plan de ses échanges épistolaires, elle ne constitue pas l'activité majeure pour laquelle Fermat revendique une supériorité propre. Il insiste au contraire à plusieurs reprises sur la démonstration des fondements utilisés. Ainsi, la caractérisation, cruciale pour le problème qui nous intéresse, des nombres premiers qui sont des sommes de deux carrés (ou encore des hypoténuses de triangles rectangles) est présentée ailleurs comme une illustration importante de la méthode démonstrative propre à Fermat, la descente infinie ${ }^{48}$. Comme nous allons le voir maintenant, les priorités de Frenicle sont tout autres.

46. Fermat, $O C$, vol. II, p. 216. On notera que si la lettre est écrite à Mersenne, les explications condensées sont adressées à Frenicle.

47. Voir, p. ex., le résumé qu'il envoie à Pascal en 1654, in Fermat, $O C$, vol. II, p. 312313. Son autre grand centre d'intérêt concerne les équations diophantiennes en nombres rationnels.

48. Voir la lettre à Pierre de Carcavi de 1659, in Fermat, $O C$, vol. II, p. 435-436. Weil, 1984, chap. II, explicite une preuve par descente, plus tardive, de ce résultat. Sur le rôle de la descente infinie chez Fermat, voir ITARD, 1950 et Goldstein, 1993. 
Le point de vue de Frenicle nous est en partie transmis dans le dernier chapitre de son Discours des triangles rectangles en nombres; nous ne connaissons pas la date exacte de sa composition, mais même si le Discours n'a jamais été publié, il constitue un texte abouti, et non des notes personnelles éparses. Le dernier chapitre traite tout entier de la construction d'ellipses possédant certains segments rationnels (en fait entiers), d'abord trois (les deux diamètres et la distance focale), puis quatre (c'est «notre » problème des ellipses), puis cinq (avec divers segments). Il est étroitement lié à l'ensemble du Discours, en ce que le cadre des triangles rectangles en nombres y est opératoire d'un bout à l'autre : la géométrie de l'ellipse est immédiatement traduite en termes de triangles, la construction d'une ellipse avec nos quatre segments entiers s'exprimant ainsi comme celle de deux triangles rectangles en nombres ayant un côté commun et tels que l'hypoténuse de l'un soit somme de l'hypoténuse de l'autre et du troisième côté (on y reconnaîtra facilement les triangles de côtés égaux à $A B, C D$, $F F$ ' et $H F, H F$ ' et $F F^{\prime}$ respectivement) ; c'est même sans référence explicite à des triangles associés que Frenicle déploie directement pour certains segments de l'ellipse des notions élaborées auparavant pour les côtés de triangles.

Contrairement à ses correspondants, par ailleurs, Frenicle ne semble pas privilégier une portion particulière du problème qui contiendrait pour lui l'essentiel de la difficulté : une variété d'aspects et d'endroits spécifiques de la résolution sont coordonnés, qui relèvent chacun d'heuristiques, d'approches, d'épreuves propres. N'est pas seul en jeu l'écart attendu entre un traité détaillé et des esquisses de réponses lancées au cours d'un échange épistolaire : Frenicle, au contraire, épouse au plus près les formes privilégiées dans la correspondance et son mode de rédaction n'est pas celui d'un traité euclidien, ni même celui, pourtant plus informel, du schéma de solution que j'ai proposé plus haut. Je me concentrerai dans ce qui suit sur deux aspects principaux : l'activation et l'articulation dans le texte de différents registres de l'expérience de Frenicle; la perception, du côté de Frenicle cette fois, de l'argumentaire des algébristes.

\section{a) Trois modes d'expérience}

J'utilise ici le mot «expérience » dans la multiplicité actuelle, courante, de ses acceptions : pratique que l'on a de quelque chose, acquisition intel- 
lectuelle ou spirituelle résultant de l'exercice des facultés au contact de la $v^{4}{ }^{49}$. Plusieurs historiens des sciences ou des idées se sont attachés à cerner le sens de ce mot, et celui de son frère ennemi, « expérimentation », dans les textes du XVI et du XVII ${ }^{e}$ siècle, à analyser la répartition et le recouvrement de leurs champs sémantiques, leurs évolutions et leurs usages, afin de comprendre l'avènement de l'expérimentation comme composante cruciale du travail scientifique moderne ${ }^{50}$. Mon objectif est tout différent, car je m'intéresse ici à l'ensemble des résonances du mot «expérience »: il s'agit de décrire comment, dans le contexte d'un écrit sur le problème sur les ellipses, sont mathématiquement activés et coordonnés des fragments de la vie de Frenicle provenant de sphères a priori distinctes (apprentissage, état de l'arithmétique au $\mathrm{XVII}^{\mathrm{e}}$ siècle, réseaux de sociabilité $)^{51}$.

Déjà évoquée, c'est la continuité entre la forme du Discours et le fonctionnement de la correspondance qui frappe tout d'abord. Les explications données par Frenicle privilégient, comme dans les lettres, les règles expliquées sur un exemple numérique. De plus, seuls les problèmes sont mis en évidence matériellement dans le texte, par le mot «Problesme» en marge; les autres types d'énoncés ne sont pas distingués spécialement. Il semble souvent s'agir moins de résoudre un problème que de le fabriquer. Frenicle décrit différentes situations, des agencements de segments ou de nombres, puis les transforme en un problème : «On pourra donc proposer ce qui s'y s'ensuit. » Cette structure évoque la promesse de la préface aux Préludes à l'Harmonie universelle que Mersenne adressait à M. de Bourges en 1634 : «La neuvième question vous fournira d'idées pour examiner les plus savants analystes qui se vantent de résoudre toutes sortes de problèmes numériques $^{52}$. $\gg$ Le texte de Frenicle se présente ainsi comme un déplacement de l'auteur au milieu de sujets familiers, pour les mobiliser en vue d'une pratique collective spécifique.

Cette impression de familiarité privée, issue d'une expérience personnelle du mathématicien, est provoquée à chaque instant par l'apparition soudaine dans le texte d'informations nécessaires à la résolution des problèmes, mais qui n'ont été ni postulées auparavant, ni établies par un déroulement systématique de l'argumentation, et qui parfois même semblent le contredire. Considérons par exemple la construction du diamètre commun aux ellipses cherchées : le ressort en est ici la relation $2 H F$. $A B=C D^{2}$. Frenicle en déduit que « $H F$ doit être égale a un quarré ou mul-

49. Je paraphrase ici les dictionnaires de français usuels, en part. Le Petit Robert.

50. Voir, p. ex., Clarke, 1982, chap. iI ; Shapin et Schaffer, 1985, chap. IV, surtout p. $127-$ 131; EAmon, 1994, p. 210-217; Dear, 1995, en part., p. 21-27 et 55-58.

51. Un projet analogue, sur un corpus de nature et de taille très différentes, est décrit par Simona Cerutti, in CerutTi, 1996, voir, en part., ses remarques sur la reconstitution de l'expérience individuelle, p. 178-184, passim.

52. Mersenne, Correspondance, vol. IV, p. 213. 
tiple d'un quarré \& lors la ligne $A K$ est pareillement un quarré ou multiple d'un quarré ${ }^{53} »-$ c'est le «pareillement » qui porte ici l'information : $A K$ (moitié de $A B$ ) est un carré si $H F$ l'est, et plus généralement le même multiple d'un carré que $H F$. Le problème de la parité du diamètre est tout d'abord négligé et Frenicle semble même oublier instantanément le cas où un multiple différent de 1 intervient, vérifiant en revanche longuement que lorsque $A B$ et $2 H F$ sont des carrés, la relation $2 H F$. $A B=C D^{2}$ est possible. Or, tous les facteurs négligés réapparaissent à l'occasion, lorsqu'ils deviennent nécessaires à la construction ou à l'énumération. Après avoir ainsi annoncé que le grand diamètre doit être le carré d'une hypoténuse, Frenicle ajoute un «Nota qu'il faut toujours prendre le plus grand quarré impair qui puisse diviser le nombre s'il n'est point quarré ${ }^{54} »$, et indique alors dans le cas général comment calculer le nombre d'ellipses associé à chaque diamètre (ce qui dépendra bien de la parité du diamètre). La mâ̂trise effective de la situation globale n'est donc pas en défaut, même si la rédaction ne la transmet que par bribes, apparemment contradictoires, sans réussir (ou chercher) jamais à l'articuler en une structure déductive complète.

Si les commentaires sont souvent assez détaillés pour que nous y percevions facilement l'embryon d'une démonstration possible, ce ne sont donc ni des syllogismes, ni des implications systématiquement conduites qui ordonnent l'exposé ou la recherche. L'heuristique de Frenicle ne prend pas plus appui sur la succession d'étapes que peut suggérer l'argumentation même, c'est-à-dire sur une analyse logique, que sur une analyse algébrique. En revanche, elle s'acquiert en partie par expérience, cette fois dans le sens plus technique d'une expérimentation mathématique contrôlée; nous pouvons la voir à l'œuvre pour la détermination et le dénombrement des hypoténuses, non dans le dernier chapitre du Discours, mais dans la Méthode des exclusions qui expose cette question comme quatrième exemple ${ }^{55}$. Frenicle a construit à l'occasion des exemples précédents une table systématique des nombres hypoténuses de triangles rectangles :

« Je trouve dans ma table quelques nombres composés comme $25,65,85 \&$ je trouve que 25 ne sert qu'a un seul triangle primitif non plus que les nombres premiers; mais que $65 \& 85$ servent chacun à deux triangles primitifs. Il faut donc qu'il y ait quelque ressemblance entre 25 et les nombres premiers qui ne soit pas entre 65 et $85 \&$ lesdits nombres premiers ${ }^{56}$. »

53. Discours, $4^{\mathrm{e}}$ cahier, p. 73.

54. Discours, $4^{\mathrm{e}}$ cahier, p. 78.

55. Pour une étude de cette Méthode et de ses relations avec des formes contemporaines d'expérimentation, voir ma communication « Baconian mathematics », présentée en mai 1998 au séminaire de Lorraine Daston, Max-Planck Institut für Wissenschaftsgeschichte und -Theorie, Berlin.

56. Frenicle, 1729, p. 27. 
L'hypothèse de Frenicle est que le phénomène est lié à ce que 25, contrairement à 65 ou à 85 , contient un unique facteur premier; cette hypothèse est alors testée sur la table et confirmée : «[...] d'où je conclus la vérité $\mathrm{du}$ dit théorème ${ }^{57}$. » On est ici très proche d'une conception de la vérité comme probabilité, tel que la décrit Bernard Rochot pour Pierre Gassendi : « [...] chose qui pour nous, pour notre pensée et notre action a valeur de vérité ${ }^{58} » ;$ la vérité est éprouvée, par et dans l'action, une action qui lie le mathématicien au domaine numérique par la construction, le repérage dans des tables, le calcul. Dans d'autres exemples, une première hypothèse est infirmée : l'expérience ne joue donc pas seulement un rôle rhétorique, mais bien un rôle heuristique. Après un examen supplémentaire, Frenicle constate qu' « un nombre qui ne se mesure que par un seul nombre premier ne sert d'hypoténuse primitive qu'à un seul triangle. S'il se mesure par deux nombres, il sert à deux triangles. S'il se mesure par trois nombres premiers, il sert à quatre triangles ${ }^{59} »$; il cherche alors à établir entre les deux suites de nombres qui interviennent (1.2.3. ... et 1. 2. 4. ...) une correspondance régulière, qu'il confirme cette fois par un argument combinatoire. Puisque « la multitude des triangles ausquels un nombre sert d'hypoténuse n'augmentent pas pour la grandeur des parties, mais seulement pour leur multitude ${ }^{60} »$ (autrement dit le nombre de triangles ne dépend que du nombre de facteurs et de leurs exposants, pas de leur valeur absolue), Frenicle code les facteurs premiers du nombre par des symboles algébriques pour achever le décompte. Une démarche analogue, bien que plus compliquée, lui indique que les nombres premiers qui sont des hypoténuses sont ceux de la forme $4 n+1$. Cette panoplie de procédés, au service d'une heuristique expérimentale, se retrouve d'ailleurs dans d'autres ouvrages de Frenicle, comme l'Abrégé des combinaisons ${ }^{61}$.

C'est ainsi d'une triple expérience que résulte la conviction. Si l'expérimentation sur des tables et des nombres participe à la construction de l'expérience personnelle du mathématicien, la réciproque est tout aussi vraie : mémoire, acuité du regard et du discernement sont explicitement sollicitées lors de la phase exploratoire. Comme le souligne l'abondance des verbes actifs à la première personne du singulier ( « je considère », « je regarde », « je cherche », «j'éprouve»), le mathématicien selon Frenicle, loin de se contenter de besogner, comme l'insinuent les analystes, doit aiguiser ses sens et son entendement, repérer des analogies, les interpréter,

57. Frenicle, 1729, p. 27.

58. Centre international de synthèse, 1955, p. 76.

59. FReNiCle, 1729, p. 29.

60. Frenicle, 1729, p. 31.

61. Coumet, [1968], vol. I, p. 155 et vol. II, p. 467-470. 
les tester. Mais sa conviction privée est articulée en aval de la découverte à l'épreuve même que constitue l'obtention d'une solution numérique valide. Comme Frenicle lui-même le remarque à propos de sa méthode : «Cette recherche ne sert principalement qu'aux questions possibles [...] ne se servant pour la plupart d'autre démonstration que de la construction ${ }^{62}$. »Une fois la construction obtenue, la collectivité tout entière peut faire sa propre expérience de validation, qui se réduit cette fois à vérifier si la solution est exacte. «En matière de problesmes, dit Descartes à une autre occasion, c'est assez d'en donner le facit, puis ceux qui l'ont proposé peuvent examiner s'il est bien résolu ou non ${ }^{63}$. » À la capacité de poser et de résoudre des problèmes comme objectif majeur de l'activité mathématique s'ajuste une certitude commune fondée principalement sur la présentation d'un objet solution.

\section{b) Les objections des algébristes du côté de Frenicle}

Qu'en est-il donc des principales réserves des algébristes ${ }^{64}$ ? Au début de sa Méthode des exclusions, Frenicle annonce: «Quoique les questions doivent estre examinées diversement selon la diversité de leur sujet, on peut néanmoins y observer quelques règles qui conviennent à toutes en

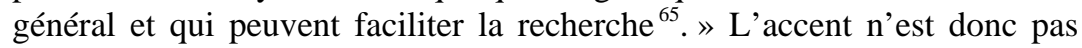
mis sur l'universalité, éventuellement comprise en un sens différent des algébristes, mais sur la résolution effective des problèmes particuliers : c'est la méthode d'exploration qui crée l'unité.

Mais si les procédures de résolution de Frenicle ne sont expliquées que sur des exemples numériques, ceux-ci sont souvent sollicités contre leur singularité, dans le sens d'une systématisation maximale. Partant d'une ellipse de grand diamètre 50 et de petit diamètre 40 , Frenicle construit ainsi $H F$ en commentant : «Je prends le quarré de 4 savoir 16 [...] ce 4 est le côté de 3. 4. 5. qui a raport a $40^{66}$. » Il s'agit bien de garder trace de l'ensemble des relations générales (arithmétiques) entre les segments considérés, toujours disponibles, semble-t-il, sous la donnée concrète des

62. Frenicle, 1729, p. 10.

63. Descartes, $O C$, vol. II, p. 94.

64. Je n'aborderai pas ici une autre objection importante, la question de l'utilité de ces problèmes, qui exigerait une étude séparée. «On ne peut traiter d'inutile en science l'acquisition d'aucune vérité », rétorque Frenicle lorsque John Wallis se plaint lui aussi de problèmes sur les nombres en 1657. La note manuscrite de Mersenne sur le problème des ellipses se trouve justement au chapitre sur «L'utilité de l'harmonie » de l'Harmonie universelle qui insiste sur le perfectionnement de l'entendement. Sur la notion d'utilité en sciences à la Renaissance et au début du XVII ${ }^{\mathrm{e}}$ siècle, voir plus généralement HAHN, 1971, CifOLETTI, 1986, et STURDY, 1995.

65. Frenicle, 1729, p. 3.

66. Discours, $4^{\mathrm{e}}$ cahier, p. 76. 
nombres utilisés. Pour une autre ellipse, de diamètres 25 et 20, Frenicle calcule $H F$ à l'aide de la relation $2 H F . A B=C D^{2}$, soit 8 , et $H F$ ' à partir de la propriété caractéristique de l'ellipse appliquée au point $H, H F$ ' $=A B-H F$, soit 17. Il commente pourtant: "Cette hipotenuse $H F$ ' est toujours la somme de deux [puissances quatrièmes] qui ont pour racines les quarrés dont 5 racine de $A B$ est la somme ${ }^{67} \gg$, une information que la voie privilégiée pour le calcul ne met pas en évidence, mais qui rend explicite le degré de généralité ${ }^{68}$ d'une apparente particularité numérique de l'exemple considéré. Celui-ci peut même être utilisé contre l'évidence numérique : trouvant à partir d'un triangle 8. 15. 17. une ellipse de distance focale 510 et de petit diamètre 272 , Frenicle remarque : «Si la distance des points brulants etoit moindre que le petit diametre, il faudrait prendre le petit coté 8 . au lieu de $15^{69}$.»

Par ailleurs, comme nous l'avons déjà remarqué, Frenicle ne se dérobe pas à l'exigence d'une démarche méthodique, qui garantit pour lui l'accès à la vérité en affinant et guidant le raisonnement : alors que le fonctionnement des échanges autoriserait aisément un pur déploiement de virtuosité extravagante, avec des nombres immenses accommodés en problèmes, comme le craignent les analystes, Frenicle souhaite obtenir des règles certaines, ne pas procéder à tâtons. Cette exigence opère clairement à propos d'une des conditions supplémentaires imposées aux ellipses solutions : que la distance focale soit supérieure au petit diamètre. En effet, si le diamètre est impair, nous l'avons vu et Frenicle l'illustre numériquement, une seule des deux ellipses associées au même triangle rectangle a pour mesure de $H F$ un entier; il arrive qu'elle vérifie la condition supplémentaire indiquée, mais pas toujours. Exprimant cette condition sur le triangle rectangle associé, Frenicle commente: «Donner une regle certaine pour Trouver un nombre qui serve d'hypoténuse a tant de triangles qu'on voudra $\&$ non plus chaqu'une desquels ayt son moindre coté impair. Il seroit difficile ${ }^{70}$. » En revanche, dans le cas où toutes les ellipses ont un $H F$ entier, c'est-à-dire si le grand diamètre est pair, une ellipse sur deux exactement - sans qu'on puisse prédire a priori laquelle - vérifiera la condition cherchée et il devient possible de maîtriser le comportement générique dans le cadre d'un dénombrement. «Si on veut qu'en toutes les Ellipses, écrit Frenicle dans le Discours, le petit diamètre soit moindre que la distance des foyers, on ne

67. Discours, $4^{\mathrm{e}}$ cahier, p. 75 .

68. Lorsque $w$ est comme dans cet exemple hypoténuse d'un triangle rectangle primitif, c'est-à-dire dont les côtés sont premiers entre eux, elle peut être écrite comme somme de deux carrés $w=r^{2}+s^{2}$, les formules données plus haut (p. 434) montrant alors facilement que $H F^{\prime}=r^{4}+s^{4}$.

69. Discours, $4^{\mathrm{e}}$ cahier, p. 83.

70. Discours, $4^{\mathrm{e}}$ cahier, p. 79 . 
pouroit y satisfaire prenant un quarré impair pour diamètre, si ce n'est par hazart $^{71}$. » Cette perspective permet de comprendre une remarque de Frenicle à Descartes que celui-ci rapporte ainsi : «Il dit que si $D C$ est un quarré impair, il ne pourra servir à aulcune ellipse dont les lignes requises s'expriment par nombres entiers $[\ldots]^{72} \gg$ (ce sur quoi Descartes en exhibe immédiatement une) : Frenicle ne vise pas du tout le comportement individuel d'une ellipse solution, mais bien la systématique de leur construction d'ensemble.

Le dénombrement joue donc un rôle clé dans la pratique arithmétique de Frenicle. Bien que le titre du chapitre évoque les «lignes rationnelles » (c'est-à-dire en proportion rationnelle avec le diamètre), seuls des exemples avec des segments entiers sont étudiés, après les premières pages. Le passage de rationnels à entiers est ainsi commenté : «Il faut prendre garde que la ligne qui sert de grand diamètre ne peut etre autre qu'un nombre entier, à cause de la clause $\&$ non plus ${ }^{73}$. » Dès qu'on admet toutes les solutions fractionnaires, l'intérêt et la possibilité même du dénombrement disparaît : il existe par exemple une infinité de triangles rectangles à côtés rationnels d'hypoténuse (même entière) donnée. Cette articulation entre dénombrement, finitude et problèmes sur les entiers, est cruciale dans la perspective d'un contrôle effectif complet des solutions. La mise en évidence et l'examen exhaustif des cas à étudier relèvent ainsi chez Frenicle plus d'une combinatoire que d'une logique ${ }^{74}$. La combinatoire requiert du coup la plus grande attention, elle qui fournit à la fois un type d'énoncés et une procédure d'analyse pour répondre à une des principales critiques des algébristes : contrôlant la régularisation des exemples numériques singuliers en prenant en compte leur comportement d'ensemble, elle permet de substituer au tâtonnement une règle certaine.

Qu'en est-il de la deuxième critique, qui porte sur le caractère lent et fastidieux de la besogne numérique? Dans la mesure où une solution numérique explicite est demandée, la gestion du temps joue pour Frenicle comme pour ses contemporains un rôle stratégique, parce qu'elle discrimine, entre les méthodes, la plus efficace. Certains énoncés proposés par Frenicle incluent d'ailleurs explicitement le temps et dans la Méthode des exclusions, Frenicle insiste sur le moyen de «faire la perquisition courte $^{75} \gg$. Le même thème apparaît chez Mersenne dans ses Harmonico-

71. Discours, $4^{\mathrm{e}}$ cahier, p. 79.

72. Descartes, $O C$, vol. II, p. 537. Les éditeurs de Descartes, à juste titre sceptiques devant une erreur aussi grossière de Frenicle, concluent à un malentendu (p. 541).

73. Discours, $4^{\mathrm{e}}$ cahier, p. 71.

74. Je rejoins ici la proposition de Coumet, [1968], vol. II, p. 499, souhaitant prendre au pied de la lettre le mot « analyse » dans « analyse combinatoire ».

75. FRENICLE, 1729, p. 6. 
rum Libri, à propos de dénombrements ${ }^{76}$. Il ne s'agit plus alors d'éliminer le hasard, mais de restreindre son emprise sur le travail mathématique en raccourcissant la route vers la solution. À plusieurs reprises, Frenicle décrit différents modes d'accès aux solutions, dont la rapidité dépend du travail déjà effectué auparavant; l'expérience accumulée dans le passé intervient donc directement dans l'efficacité d'une procédure ${ }^{77}$. Dans le Discours, la vitesse avec laquelle on peut atteindre les solutions ramène aussi de manière instructive à la question de l'algèbre. Par exemple, pour déterminer deux segments, Frenicle est amené à «partir 70 en deux telles parties que le quarré de l'une etant oté de celuy de l'autre il reste 2500 [...]. Ce qui se fera par la voye ordinaire posant pour une partie un nombre incognu \& partant l'autre partie sera 70 moins ce nombre incognu \& poursuivant la règle on trouvera $[\ldots]^{78} \gg$. Frenicle n'ignore donc pas complètement l'algèbre de Jérôme Cardan ou de Diophante. Mais dans ces propres exemples, elle peut être souvent remplacée par une procédure plus rapide : «[Le segment cherché] se pourra trouver autrement sans suposer de nombre incognu ${ }^{79}$. » L'algèbre peut être mise en œuvre en tant que règle, les symboles algébriques peuvent intervenir, nous l'avons vu plus haut, en tant que code, mais l'analyse algébrique semble à Frenicle peu efficace comme point d'appui d'une pratique mathématique : «Je sais, écrit-il à Fermat, que l'Algèbre de ce pays-ci n'est point propre pour soudre ces questions ${ }^{80}$. »

\section{L’EXPÉRIENCE DES NOMBRES DE FRENICLE DE BESSY}

«Les hommes ne sont pas dans des catégories sociales comme des billes dans des boîtes, [...] les "boîtes" n'ont d'autre existence que celle que les hommes [...], en contexte, leur donnent. » «Au point de départ d'un nouveau moment historiographique », Bernard Lepetit ${ }^{81}$ proposait donc en particulier de considérer que «les identités sociales ou les liens sociaux n'ont pas de nature, mais seulement des usages ». Un tel constat, s'il est tout aussi pertinent pour les identités sociales investies dans une activité scientifique, ne définit pourtant pas une unique voie de recherche. Suivant des

76. Coumet, [1968], vol. I, p. 121.

77. Des préoccupations analogues sont réapparues récemment en informatique théorique, dans le contexte de l'estimation du temps de calcul et de la complexité algorithmique.

78. Discours, $4^{\mathrm{e}}$ cahier, p. 89.

79. Discours, $4^{\mathrm{e}}$ cahier, p. 89.

80. Fermat, $O C$, vol. II, p. 227.

81. LePETIT, 1995, p. 13. 
suggestions de l'anthropologie interprétative, on peut considérer ces caractérisations sociales comme des représentations, la tâche principale étant alors de comprendre comment elles se prêtent à l'emploi scientifique, par exemple en mobilisant de nouveaux publics ou en favorisant certaines formes textuelles. Mais si on se focalise sur l'examen du travail lui-même, l'accent tend à se déplacer vers le pluriel du mot « usages » : ce qui retient alors l'attention, c'est la dissociation d'identités apparentes que ces usages produisent ou mettent en lumière. Non le mode d'idéalisation de leurs registres agglomérés dans l'élaboration d'une société savante, mais la reconstitution du mode opératoire de chacun d'eux dans l'élaboration des mathématiques. C'est cette seconde voie que j'ai suivie ici.

Je n'ai donc pas cherché à restituer la cohérence globale de la personnalité de Frenicle, ni à déterminer de quel modèle social il se faisait, même tacitement, le porte-parole, mais plutôt à situer, ancrées dans ses pratiques, les apparentes oppositions dont il paraissait traversé. Docte honnête homme ${ }^{82}$, Frenicle n'incarne pourtant ni «l'» honnête homme, ni «le» docte. S'il peut être perçu comme dogmatique par les algébristes dont il juge avec véhémence les solutions incomplètes, ce n'est pas au nom d'une exigence théorique scolastique ou d'un langage universel: dogmatique dans l'échange, Frenicle ne l'est pas dans la démarche. Certains de ses contemporains eurent à cœur d'ajuster étroitement les usages civil et disciplinaire du mot «dogmatique », fustigeant l'outrance pédante de la certitude mathématique, louant à rebours la modestie courtoise de l'observation singulière : l'exemple de Frenicle témoigne que cet ajustement n'allait pas de soi. Si de même Frenicle calcule de grands nombres — sa plus petite solution pour le problème de 12 ellipses, rapportée par Mersenne, comporte 17 chiffres - , ce n'est pas en curieux fasciné par la singularité, l'immense, l'étrange : ces nombres sont là pour porter témoignage de l'efficacité de sa méthode, les uns garantissant l'autre et réciproquement. Entre curioso et universitaire, Frenicle ouvre une brèche qui permet au moins d'entrevoir la complexité concrète des activités mathématiques dans la France du premier XVII siècle.

Comme dans d'autres circonstances analogues, lors de la redécouverte d'une culture, d'un personnage, d'un texte, d'un instrument, nous sommes tentés non seulement de compléter les anciens chantiers, mais d'en ouvrir de nouveaux. Frenicle plaide ainsi pour une histoire de l'heuristique, une histoire de la conviction, une histoire de la notion de «résultat» et de «solution» - en particulier une histoire de l'effectivité en mathéma-

82. Sur l'opposition classique entre honnête homme et docte, voir MAgENDIE, 1925, BIAGIOLI, 1993, p. 112-120, et MAZAURIC, 1997, p. 80 sq.; sur la version anglaise de cette opposition, et l'éventuelle réconciliation du gentleman et du scholar, voir Shapin, 1991. Les limites, de territoires et d'expressions, de la civilité, sont discutées in DASTON, 1991. 
tiques - , une histoire des modes de classification des savoirs. Je me limiterai ici à mentionner deux questions, circonscrites à Frenicle: l'une concerne son succès, l'autre son échec. Son succès, d'abord, c'est-à-dire sa place dans le cercle mersennien, que j'ai surtout exposée ici dans ses aspects polémiques et dont il importerait de préciser la configuration globale. Comme le montre la question des ellipses, l'opposition à Descartes ne s'est pas manifestée seulement sur des questions de philosophie naturelle; plusieurs décennies avant le projet leibnizien, une orientation combinatoire a bien constitué une alternative possible, ou au moins une pierre d'achoppement, aux propositions cartésiennes.

Quant à l'échec de Frenicle, c'est-à-dire son effacement historique, est-il seulement celui de l'historiographie pour n'avoir pu prendre en compte son projet en ses termes propres? Les remaniements du Discours des triangles rectangles dès la mort de son auteur soulignent au contraire l'embarras de ses successeurs immédiats, leur volonté de restructurer les propositions de Frenicle dans le cadre plus traditionnel d'un traité euclidien. Nous avons rouvert plusieurs fois dans les dernières décennies de multiples dossiers liés à la Révolution scientifique, ceux qui touchent à la mécanique, à l'astronomie, à la philosophie naturelle, bref à ces champs que nous percevons à tort ou à raison en amont de la physique et de la chimie modernes. Sensibles aux secousses et aux polémiques autour des nouvelles validations expérimentales, nous avons peut-être admis trop vite qu'elles ne concernaient que les champs où ces modes d'épreuves se sont au moins en partie imposés durablement. Frenicle témoigne cependant que les mathématiques théoriques elles-mêmes furent traversées par des vents analogues et nous suggère ainsi de réviser une fois de plus, mais dans une perspective nouvelle, l'opposition banale entre sciences mathématiques et sciences expérimentales et son rôle dans la dynamique des sciences modernes.

L'épisode dont je viens d'esquisser les grandes lignes ne relève pas de l'histoire des controverses. Il s'agit bel et bien d'un récit d'expérience : celle que constitua pour Frenicle le problème des ellipses dans la société mathématique à laquelle il participait. Une expérience que le genre du Discours laisse affleurer et qui se décline en plusieurs registres articulés, des mathématiques au mathématicien, de la collectivité aux mathématiques. Le temps réel du travail humain, l'affinement de l'observation, la régularisation du monde des nombres y jouent un rôle constitutif que le mode de rédaction ne cherche pas à ignorer. Les efforts passés - voire, puisque la méthode est aussi un entraînement, l'éducation passée du mathématicien sont nécessaires pour repérer les régularités utilisables; l'activité est orientée explicitement vers ce qui est nécessaire pour faire fonctionner au mieux le jeu des échanges épistolaires, dont elle retient et retourne les injonctions à faire vite, à faire certain, à faire subtil. Comme Frenicle lui-même, c'est à 
la question de retracer et de transmettre une expérience que ses historiens sont confrontés, l'expérience d'un mathématicien dont le savoir s'exprime comme un savoir-faire, la vérité comme une démarche, la méthode comme un voyage.

Catherine GoldsTEIN (septembre 2000). 


\section{LISTE DES RÉFÉRENCES}

BeAulieu (Armand), 1982, « Joutes ou combats? Italiens et Français devant le problème de la cycloïde », Recherches sur le dix-septième siècle, 5, p. 31-35.

Biagioli (Mario), 1993, Galileo courtier, Chicago, Londres, Chicago University Press.

Bos (Henk), 1996, « Tradition and modernity in early modern mathematics. Viète, Descartes and Fermat», in Catherine Goldstein, Jeremy Gray et Jim RitTer, éd., L'Europe mathématique. Histoires, mythes, identités, Paris, Éd. de la Maison des sciences de l'homme, p. 183-204.

CANTOR (Moritz), 1880-1908, Vorlesungen über Geschichte der Mathematik, Leipzig, Teubner, 4 vol.

Centre international de synthèse, 1955, Pierre Gassendi, sa vie et son æuuvre (15921655), Paris, Albin Michel.

Cerutti (Simona), 1996, « Processus et expérience. Individus, groupes et identités à Turin, au XVII ${ }^{\mathrm{e}}$ siècle », in Revel, 1996, p. 161-186.

Cifoletti (Giovanna), 1986, «L'utilité des mathématiques selon La Ramée. Brèves notes », Revue des sciences philosophiques et théologiques, t. LXX, 1, p. 99100.

Clarke (Desmond), 1982, Descartes' philosophy of science, Manchester, Manchester University Press.

Coumet (Ernest), [1968], Mersenne, Frenicle et l'élaboration de l'analyse combinatoire dans la première moitié $d u$ XVII ${ }^{e}$ siècle, thèse de troisième cycle, dacty$\operatorname{logr} ., 2$ vol., 590 p. Édition à paraître à Paris, Albin Michel (Bibliothèque de l'Evolution de l'humanité).

Coumet (E.), 1987, «Alexandre Koyré. La Révolution scientifique introuvable? », in «Science. The renaissance of history. Proceedings of the international conference Alexandre Koyré, Paris, Collège de France, 10-14 june 1986 », éd. Pietro Redondi, $\mathrm{n}^{\circ}$ spéc. History and Technology, vol. IV, 1-4, p. 497-529.

Daston (Lorraine), 1991, «Baconian facts, academic civility and the prehistory of objectivity », Annals of Scholarship, 8, p. 337-363.

Dear (Peter), 1995, Discipline and experience, Chicago, Londres, Chicago University Press.

Descartes (René), OC, Euvres complètes, éd. Charles Adam et Paul Tannery, Paris, Cerf, 1897-1913, 13 vol.

Eamon (William), 1994, Science and the secrets of nature, Princeton, Princeton University Press.

Fermat (Pierre), OC, Euvres complètes, éd. Charles Henry et Paul Tannery, Paris, Gauthier-Villars, 1891-1912, 4 vol., et Supplément, éd. Cornélis DE WAARD, Paris, Gauthier-Villars, 1922.

FRENICle DE Bessy (Bernard), 1729, Mémoires de l'Académie royale des sciences depuis 1666 jusqu'en 1699, t. V, Paris, Compagnie des Libraires [contient en particulier le Traité des triangles rectangles en nombres, édité en 1676 et 1677, la Méthode des exclusions et l'Abrégé des combinaisons, édités en 1693]. 
Gabbey (Alan), 1966 et 1990, «Inventaire des papiers de Roberval », dactylogr.

Goldstein (Catherine), 1993, «Preuves par descente infinie en analyse diophantienne. Programmes de travail et mises en œuvre chez Fermat, Levi, Mordell et Weil », Cahiers du séminaire d'Histoire des mathématiques de l'institut HenriPoincaré, 2/3, p. 25-49.

Goldstein (C.), 1995, Un théorème de Fermat et ses lecteurs, Saint-Denis, Presses universitaires de Vincennes.

Hahn (Roger), 1971, The Anatomy of a scientific institution. The Paris Academy of sciences, 1666-1803, Berkeley, Los Angeles, University of California Press.

HoFmann (Joseph E.), 1944, Neues über Fermats zahlentheoretische Herausforderungen von 1657, Abhandlungen der Preußischen Akademie der Wissenschaften, Jahrg. 1943, Mathematisch-naturwissenschaftliche Klasse, vol. IX, Berlin, Verlag der Akademie der Wissenschaften.

ITARD (Jean), 1950, «Les méthodes utilisées par Fermat en théorie des nombres », Revue d'histoire des sciences, t. III, p. 21-26.

JuHEL (Jean-Charles), 1994, «Le rôle des proportions dans l'évolution de l'écriture algébrique au $\mathrm{XVII}^{\mathrm{e}}$ siècle », Sciences et techniques en perspective, p. 61-158.

LEPETIT (Bernard), dir., 1995, Les Formes de l'expérience. Une autre histoire sociale, Paris, Albin Michel (L'Évolution de l'humanité).

Magendie (Maurice), 1925, La Politesse mondaine et les théories de l'honnêteté en France au XVII ${ }^{e}$ siècle de 1600 à 1660, Paris, Presses universitaires de France.

Mazauric (Simone), 1997, Savoirs et philosophie à Paris dans la première moitié $d u$ XVII ${ }^{e}$ siècle, Paris, Publications de la Sorbonne.

Mersenne (Marin), Correspondance, éd. $\mathrm{M}^{\mathrm{me}}$ Paul Tannery, Cornélis de WaArd, Bernard Rochot et Armand Beaulieu, 17 vol., 1932-1988, Paris, Centre national de la recherche scientifique (à partir du $5^{\mathrm{e}}$ volume).

Revel (Jacques), éd., 1996, Jeux d'échelles. La microanalyse à l'expérience, Paris, Seuil/Gallimard (Hautes études).

Shapin (Steven), 1991, «A scholar and a gentleman », History of Science, 29, p. 279-327.

Shapin (Steven) et Schaffer (Simon), 1985, Leviathan and the air-pump, Princeton, Princeton University Press.

Sturdy (David), 1995, Science and social status. The members of the Académie des sciences, 1666-1750, Woodbridge, Boydell Press.

WeIL (André), 1984, Number theory. An approach through history from Hammurabi to Legendre, Boston, Birkhäuser. 